05,04

\title{
Индуцированный полем магнитный переход в смешанном алюморедкоземельном гранате $\mathrm{Er}_{2} \mathrm{HoAl}_{5} \mathrm{O}_{12}$
}

\author{
(C) Е.В. Шевченко ${ }^{1}$, Е.В. Чарная 1 ฯ, Е.Н. Хазанов ${ }^{2}$, А.В. Таранов ${ }^{2}$, А.С. Бугаев ${ }^{3}$ \\ ${ }^{1}$ Санкт-Петербургский государственный университет, \\ Санкт-Петербург, Россия \\ ${ }^{2}$ Институт радиотехники и электроники РАН, \\ Москва, Россия \\ ${ }_{3}^{3}$ Московский фризико-технический институт (Государственный университет), \\ Долгопрудный, Россия \\ ฯ E-mail: charnaya@mail.ru
}

(Поступила в Редакцию 8 сентября 2016 г.)

\begin{abstract}
Проведены исследования температурной зависимости магнитной $a c$ восприимчивости монокристаллического смешанного граната $\mathrm{Er}_{2} \mathrm{HoAl}_{5} \mathrm{O}_{12}$ в диапазоне от 1.8 до $300 \mathrm{~K}$ в нулевом постоянном поле и при приложении смещающих полей до 9Т. В отсутствие постоянного магнитного поля восприимчивость следовала закону Кюри-Вейсса. Приложение постоянного поля индуцировало магнитный фазовый переход, температура которого возрастала с ростом напряженности магнитного поля. Характерный для фазового перехода максимум динамической восприимчивости не демонстрировал заметной зависимости от частоты переменного поля.

Работа выполнена при поддержке РФФИ (грант № 16-07-00181). Измерения проводились на оборудовании Ресурсного центра „Центр диагностики функциональных материалов для медицины, фармакологии и наноэлектроники“, Научный парк СПбГУ.
\end{abstract}

DOI: 10.21883/FTT.2017.04.44273.345

\section{1. Введение}

Алюморедкоземельные гранаты (АРГ) $\mathrm{RE}_{3} \mathrm{Al}_{5} \mathrm{O}_{12}$ ( $\mathrm{RE}$ - редкоземельные ионы) различного состава широко используются в лазерной технике [1]. В последнее время обсуждаются возможности применения АРГ в других прикладных областях, в частности в низкотемпературных магнитных рефрижераторах [2], что стимулирует интерес к изучению магнитных свойств гранатов. Наличие в решетке гранатов парамагнитных трехвалентных редкоземельных ионов (за исключением иттрия и лютеция) может приводить к возникновению магнитно-упорядоченных фаз при понижении температуры. В частности, в монокристаллах $\mathrm{Ho}_{3} \mathrm{Al}_{5} \mathrm{O}_{12}$ был обнаружен антиферромагнитный переход около $0.8 \mathrm{~K}$ [3]. Для $\mathrm{Tb}_{3} \mathrm{Al}_{5} \mathrm{O}_{12}$ и $\mathrm{Dy}_{3} \mathrm{Al}_{5} \mathrm{O}_{12}$ также было установлено существование антиферромагнетизма при низкой температуре [4,5]. Для чистого алюмоэрбиевого граната и смешанных гранатов $\mathrm{Er}_{x} \mathrm{Y}_{3-x} \mathrm{Al}_{5} \mathrm{O}_{12}$ с $x \geq 0.6$ в работе [6] сообщалось об обнаружении антиферромагнетизма с температурой Нееля около $50 \mathrm{mK}$ и ниже, хотя в работе [2] этот результат не был подтвержден. Низкие температуры антиферромагнитного перехода в АРГ связаны со структурой гранатов. Для кристаллов с решеткой гранатов с симметрией $I a \overline{3} d$, в которых ионы RE находятся в вершинах треугольников, образующих две подрешетки, магнитные моменты не могут быть ориентированы попарно антипараллельно [7]. Это приводит к геометрической фрустрации и многократному вырождению основного состояния. В таких системах можно ожидать не только значительного подавления антиферромагнетизма, но и формирования более сложных магнитных фаз, а также фазовых переходов, индуцированных магнитным полем. Действительно, в галлиевом гранате $\mathrm{Tb}_{3} \mathrm{Ga}_{5} \mathrm{O}_{12}$ наблюдались необычные метамагнитные явления [8], однако для АРГ известные магнитные свойства ограничиваются обычными переходами в антиферромагнитное состояние. В настоящей работе приводятся результаты исследований динамической магнитной восприимчивости в смешанном АРГ $\mathrm{Er}_{2} \mathrm{HoAl}_{5} \mathrm{O}_{12}$, проведенные с целью обнаружения неизвестных ранее особенностей магнитных свойств, индуцированных магнитным полем.

\section{2. Образцы и эксперимент}

Монокристалл $\mathrm{Er}_{2} \mathrm{HoAl}_{5} \mathrm{O}_{12}$ был выращен из расплава методом горизонтальной направленной кристаллизации в молибденовом контейнере (ВНИИСИМС, г. Александров). Образец для исследования представлял собой пластинку толщиной $0.5 \mathrm{~mm}$, вырезанную перпендикулярно кубической оси монокристалла. Масса образца равнялась $54.19 \mathrm{mg}$.

Измерения вещественной и мнимой частей $a c$ намагниченности проводились на комплексе для исследования физических свойств материалов в широком диапазоне температур и магнитных полей PPMS-9 + EverCool-II производства Quantum Design. Амплитуда переменного поля составляла $2.5 \mathrm{Oe}$. Частота изменялась от $50 \mathrm{~Hz}$ до $10 \mathrm{kHz}$. Постоянное (смещающее) поле менялось от 0 
до 9Т. Измерения проводились в диапазоне температур от 1.8 до $300 \mathrm{~K}$.

\section{3. Результаты}

Температурные зависимости вещественной части удельной магнитной восприимчивости, измеренные в нулевом магнитном поле и при приложении магнитных полей до 9Т, представлены на рис. 1. Комплексная удельная магнитная восприимчивость $\chi$ рассчитывалась по формуле $\chi=M / A \cdot m$, где $M-$ измеряемый комплексный магнитный момент образца, $A$ - амплитуда переменного поля, $m-$ масса образца. Показанные зависимости были получены на частоте переменного магнитного поля $1 \mathrm{kHz}$. Мнимая часть удельной восприимчивости была значительно меньше вещественной части и в полях, превышающих $1 \mathrm{~T}$, находилась на уровне погрешности измерений. Температурные зависимости обратной динамической магнитной восприимчивости показаны на рис. 2. Как видно из рис. 1 и 2, динамическая восприимчивость в нулевом поле возрастает с понижением температуры. Зависимость от температуры восприимчивости в нулевом поле выше $10 \mathrm{~K}$ (рис. 2) описывается законом Кюри-Вейсса $\chi=C /(T-\theta)$ с отрицательной температурой $\theta=-7.75 \mathrm{~K}$ и постоянной Кюри-Вейсса $C=0.0173 \mathrm{emu} \cdot \mathrm{K} / \mathrm{g}$. Ниже $10 \mathrm{~K}$ зависимость восприимчивости от температуры становится более сильной, чем предсказывает закон Кюри-Вейсса.

Приложение постоянного магнитного поля коренным образом изменяет температурную зависимость восприимчивости. В поле $0.5 \mathrm{~T}$ рост восприимчивости при

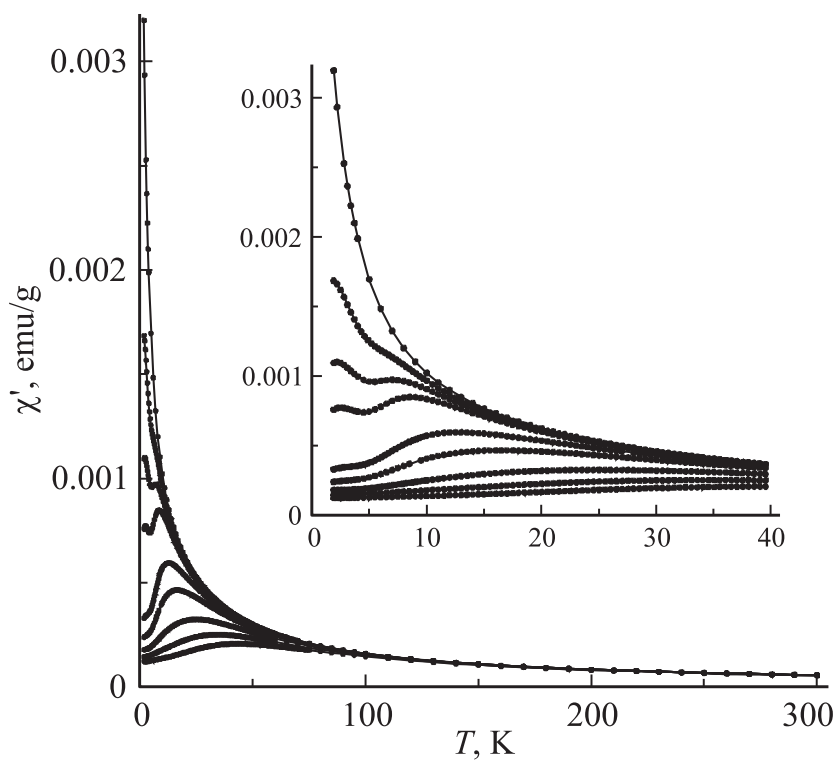

Рис. 1. Температурные зависимости вещественной части $a c$ восприимчивости $\chi^{\prime}$, измеренные без поля (верхняя кривая) и в полях $0.5,0.75,1,2,3,5,7,9$ (нижняя кривая) $T$. На вставке показаны температурные зависимости $\chi^{\prime}$ в увеличенном масштабе.

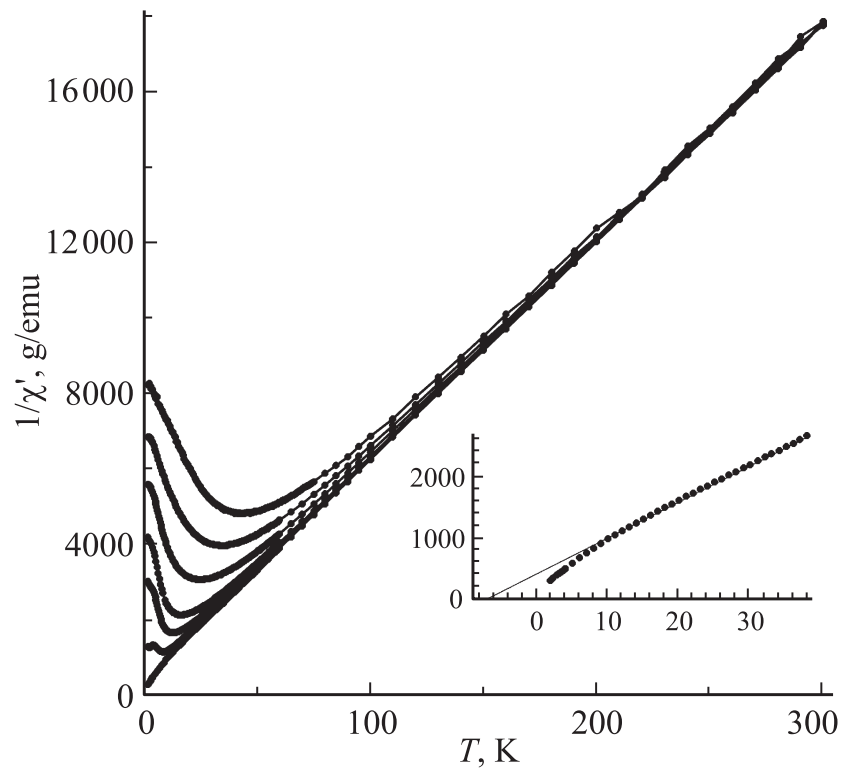

Рис. 2. Температурные зависимости обратной вещественной части $a c$ восприимчивости $1 / \chi^{\prime}$, измеренные без поля (нижняя кривая) и в полях $1,2,3,5,7,9$ (верхняя кривая) $T$. На вставке показана температурная зависимость $1 / \chi^{\prime}$ в нулевом постоянном поле в увеличенном масштабе.

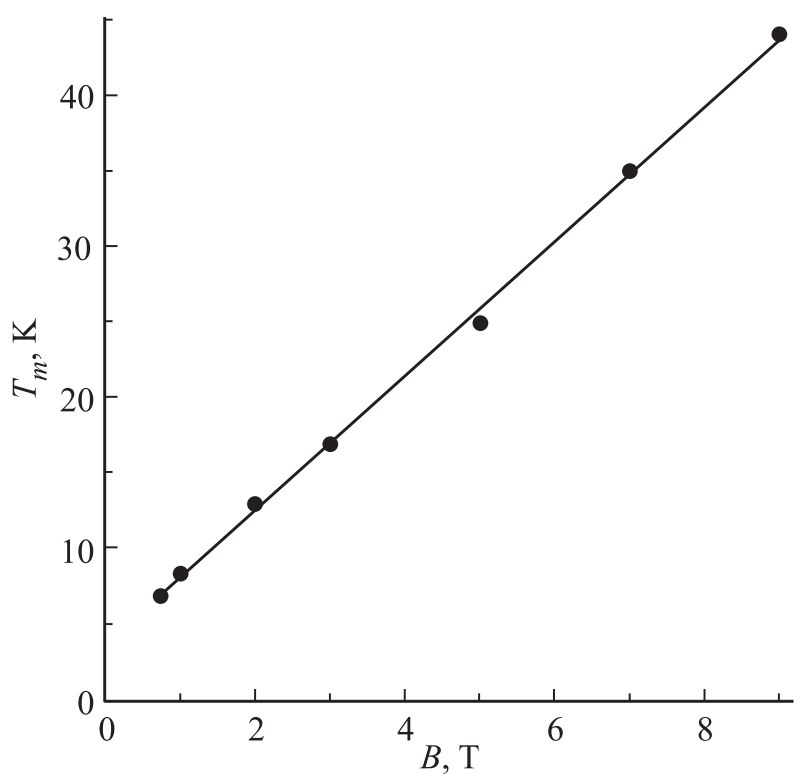

Рис. 3. Зависимость температуры максимума восприимчивости $T_{m}$ от индукции постоянного магнитного поля $B$. Прямая линия - линейная аппроксимация, полученная методом наименьших квадратов.

понижении температуры значительно ослабляется, а затем при дальнейшем возрастании поля на кривых температурной зависимости появляется локальный максимум. При еще более сильных полях ниже температуры максимума восприимчивость монотонно уменьшается. Максимум восприимчивости смещается вверх по тем- 


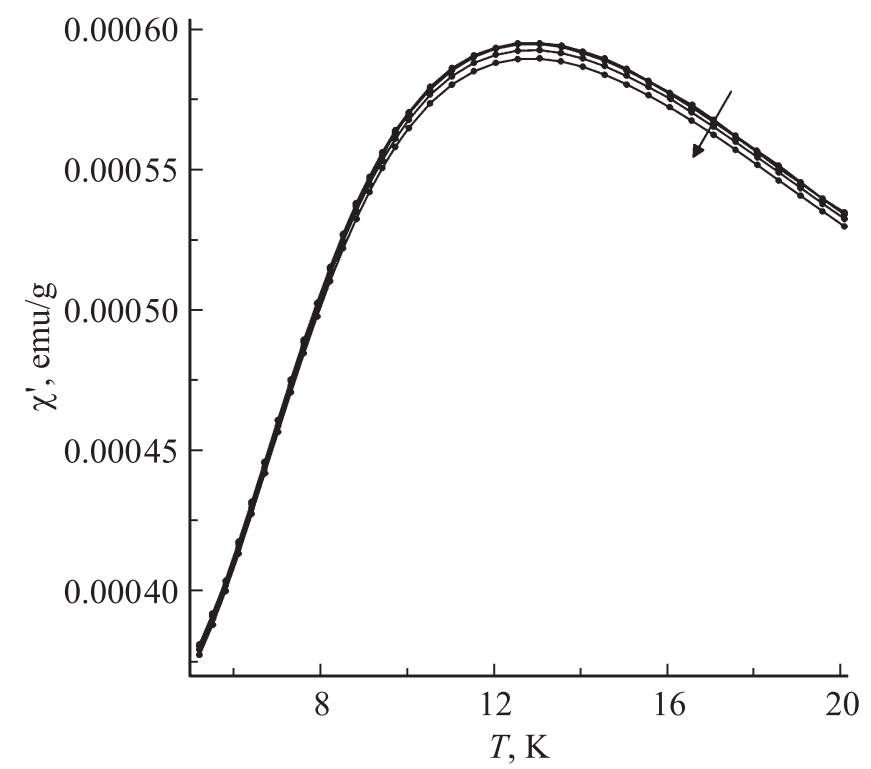

Рис. 4. Температурная зависимость $\chi^{\prime}$ вблизи максимума, измеренная на частотах $500 \mathrm{~Hz}$ и 1,5 и $10 \mathrm{kHz}$. Постоянное поле равнялось 2 Т. Стрелка показывает увеличение частоты.

пературе при увеличении поля. В поле 9 Т отклонения от закона Кюри-Вейсса становятся заметными уже при температуре около $120 \mathrm{~K}$. Зависимость температуры максимума восприимчивости $T_{m}$ от величины постоянного магнитного поля показана на рис. 3. Видно, что $T_{m}$ линейно возрастает с ростом поля.

Для выявления физической природы аномального поведения динамической восприимчивости, возникающего при приложении постоянного магнитного поля, существен характер изменения положения максимума при изменении частоты переменного поля. Наши исследования выявили отсутствие заметной частотной зависимости, что иллюстрируется для постоянного поля 2 Т на рис. 4. Показаны только данные для частот, начиная с $500 \mathrm{~Hz}$, так как на низких частотах был получен сильный разброс экспериментальных точек в пределах измерений на частотах $500 \mathrm{~Hz}$ и $10 \mathrm{kHz}$.

\section{4. Обсуждение}

Поведение вещественной части $a c$ восприимчивости в нулевом магнитном поле показывает, что смешанный гранат $\mathrm{Er}_{2} \mathrm{HoAl}_{5} \mathrm{O}_{12}$ находится в парамагнитном состоянии во всем исследованном температурном диапазоне. Усиление температурной зависимости восприимчивости ниже $10 \mathrm{~K}$ по сравнению с законом Кюри-Вейсса (см. вставку к рис. 2) может быть проявлением вклада флуктуаций при приближении к антиферромагнитному переходу с температурой Нееля, меньшей $1.8 \mathrm{~K}$. Низкая температура Нееля по сравнению с температурой Кюри-Вейсса $\theta$ отмечалась и в других АРГ [9] и обусловлена влиянием геометрической фрустрации в решетке гранатов.
Изменение температурной зависимости динамической восприимчивости при приложении достаточно сильного смещающего поля демонстрирует существование индуцированного полем магнитного фазового перехода. Максимум на кривой восприимчивости может наблюдаться как при переходах в магнитно-упорядоченное состояние, так и при переходе в фазу спинового стекла. Считается, что характерным признаком формирования спинового стекла является существенная частотная зависимость положения максимума восприимчивости. Для исследуемого нами смешанного граната заметная частотная зависимость отсутствовала, что свидетельствует в пользу установления магнитного упорядочения при приложении магнитного поля. Кроме того, при переходе в фазу спинового стекла должна наблюдаться значительная мнимая часть восприимчивости, что также не соответствует экспериментальным данным. Однако переходы в магнитно-упорядоченное состояние обычно смещаются к низким температурам при увеличении постоянного магнитного поля. Таким образом, магнитное поведение исследуемого образца является аномальным.

Индуцированные полем изменения восприимчивости, сходные с поведением намагниченности в образце $\mathrm{Er}_{2} \mathrm{HoAl}_{5} \mathrm{O}_{12}$, были обнаружены ранее для других материалов в работах $[8,10]$. В работе [8] исследовался галлиевый гранат $\mathrm{Tb}_{3} \mathrm{Ga}_{5} \mathrm{O}_{12}$. В нулевом магнитном поле температурная зависимость динамической восприимчивости соответствовала обычному парамагнетику с отрицательной температурой Кюри-Вейсса. В полях больше $1 \mathrm{~T}$ восприимчивость существенно изменялась, и на температурной кривой появлялся максимум, аналогичный показанному на рис. 1. Положение максимума смещалось к высоким температурам при увеличении постоянного магнитного поля, но смещение нелинейным образом зависело от поля. Индуцированные магнитным полем явления трактовались на основе нейтронных исследований как проявление ферромагнитных и антиферромагнитных корреляций, сосуществующих в сильных магнитных полях и связанных с изменением симметрии магнитной подрешетки. В работе [10] исследовался кристаллический $\mathrm{Co}_{2}(\mathrm{OH}) \mathrm{AsO}_{4}$. Вызванные полем изменения динамической восприимчивости значительно отличались от представленных на рис. 1, однако температуры перегиба на кривых также смещались в сторону высоких температур при усилении постоянного магнитного поля, хотя и слабее, чем в настоящей работе и в работе [8]. Полученные данные были интерпретированы как антиферромагнитный переход. Сдвиг аномалии магнитной восприимчивости в сторону высоких температур при усилении смещающего поля наблюдался также для $\mathrm{Co}_{2}(\mathrm{OH}) \mathrm{PO}_{4}$ в работе [11] и интерпретировался как переход в фазу спинового стекла. При этом указывалось, что фазовый переход в спиновое стекло может сдвигаться к высоким температурам с ростом поля и сопровождается значительными аномалиями мнимой части $a c$ восприимчивости. 
Экспериментальные результаты, полученные в настоящей работе для АРГ $\mathrm{Er}_{2} \mathrm{HoAl}_{5} \mathrm{O}_{12}$, ближе всего к результатам магнитных исследований, проведенных в работе [8]. Отсутствие частотной зависимости положения максимума восприимчивости (рис. 4) и существенных потерь в области максимума также согласуется с интерпретацией аномального поведения восприимчивости при приложении смещающего поля как следствия возникновения магнитно-упорядоченного состояния. Можно предположить, что, как и в галлиевом гранате $\mathrm{Tb}_{3} \mathrm{Ga}_{5} \mathrm{O}_{12}$, достаточно сильное постоянное магнитное поле вызывает изменение симметрии магнитной подрешетки и стабилизирует дальний магнитный порядок. При этом наличие в решетке $\mathrm{Er}_{2} \mathrm{HoAl}_{5} \mathrm{O}_{12}$ двух видов парамагнитных редкоземельных ионов, эрбия и гольмия, приводит к дополнительной фрустрации и усиливает влияние магнитного поля. Для подтверждения сделанного предположения и установления характера индуцированных полем спиновых корреляций необходимы дополнительные исследования.

Таким образом, в настоящей работе обнаружен индуцированный магнитным полем фазовый переход в магнитной подрешетке смешанного граната $\mathrm{Er}_{2} \mathrm{HoAl}_{5} \mathrm{O}_{12}$, ранее не наблюдавшийся в алюморедкоземельных гранатах. Температура характерного для фазового перехода максимума динамической восприимчивости линейно возрастает с усилением магнитного поля от 0.75 до 9Т. Предполагается, что магнитное поле стабилизирует дальний магнитный порядок и изменяет симметрию магнитной подрешетки.

\section{Список литературы}

[1] А.А. Каминский. Лазерные кристаллы. Наука, М. (1975). $356 \mathrm{c}$.

[2] A. Kushino, Y. Aoki, N.Y. Yamasaki, T. Namiki, Y. Ishisaki, T.D. Matsuda, T. Ohashi, K. Mitsuda, T. Yazawa. Erbiumdopped yttrium aluminum garnet as a magnetic refrigerant for low temperature x-ray detectors. J. Appl. Phys. 90, 5812 (2001).

[3] S. Nagata, H. Sasaki, K. Suzuki, J. Kiuchi, N. Wada. Specific heat anomaly of the holmium garnet $\mathrm{Ho}_{3} \mathrm{Al}_{5} \mathrm{O}_{12}$ at low temperature. J. Phys. Chem. Solids 62, 1123 (2001).

[4] B.E. Keen, D.P. Landau, W.P. Wolf. Magnetic transition in a multi-axis antiferromagnetic. Phys. Lett. 23, 202 (1966).

[5] D.P. Landau, B.B. Keen, B. Schneider, W.P. Wolf. Magnetic and thermal properties of dysprosium aluminum garnet. I. Experimental results for the two-sublattice phases. Phys. Rev. B 3, 2310 (1971).

[6] Х.С. Багдасаров, А.П. Додокин, А.А. Сорокин. Магнитная восприимчивость кристаллов твердых растворов $/ \mathrm{Er}_{x} \mathrm{Y}_{1-x} /{ }_{3} \mathrm{Al}_{5} \mathrm{O}_{12}$ при низких температурах. ФТТ 30, 1840 (1988).

[7] I. Mirebeau, I.N. Goncharenko, P. Cadavez-Pares, S.T. Bramwell, M.J.P. Gingras, J.S. Gardner. Pressure-induced crystallization of a spin liquid. Nature 420, 54 (2002).
[8] K. Kamazawa, D. Louca, R. Morinaga, T.J. Sato, Q. Huang, J.R.D. Copley, Y. Qiu. Field induced ferromagnetism and competition in the metamagnetic state of terbium gallium garnet. Phys. Rev. B 78, 064412 (2008).

[9] A.P. Ramirez. In: Handbook of magnetic materials/ Ed. K.H. Buschow. Elsevier, N.Y. (2001). V. 13, 423.

[10] I. de Pedro, J.M. Rojo, J. Rodríguez Fernández, M.T. Fernández-Díaz, T. Rojo. Sinusoidal magnetic structure in a threedimensional antiferromagnetic $\mathrm{Co}_{2}(\mathrm{OH}) \mathrm{AsO}_{4}$ : Incommensurate-commensurate magnetic phase transition. Phys. Rev. B 81, 134431 (2010).

[11] J.M. Rojo, J.L. Mesa, L. Lezama, J.L. Pizarro, M.I. Arriortua, J. Rodríguez Fernández, G.E. Barberis, T. Rojo. Spin-glass behavior in a three-dimensional antiferromagnet ordered phase: Magnetic structure of $\mathrm{Co}_{2}(\mathrm{OH})\left(\mathrm{PO}_{4}\right)$. Phys. Rev. B 66, 094406 (2002). 